\title{
A COMPARISON BETWEEN RADIOHELIOGRAMS AND OPTICAL OBSERVATIONS OF THE SOLAR CORONA
}

\author{
M. WALDMEIER \\ Swiss Federal Observatory, Zürich, Switzerland
}

Beginning in summer 1957, W. N. Christiansen and D. S. Mathewson [1] have regularly obtained two-dimensional radioheliograms at $\lambda=21 \mathrm{~cm}$ with a resolving power of 3 minutes of arc. The authors have already noticed the close connection between the regions of radio emission on the one side and the fields of spots and faculae on the other side. Considering, however, that the radio emission at the wavelength involved emerges from heights of 20,000 to 50,000 , or even up to $100,000 \mathrm{~km}$ above the photosphere, i.e. from the inner corona, it seems to be more suitable to compare the radioheliograms with the optical emission of the corona than with the photospheric and chromospheric phenomena. Yet, as the coronal observations can be carried out at the solar limb only, it is difficult to get optical pictures of the corona in front of the sun's disk. Such a picture has to be built up from the daily limb-observations covering the period from 7 days before to 7 days after the date in question; e.g. the coronal intensities shown along the central meridian are measured 7 days before at the E-limb or 7 days later at the $\mathrm{W}$-limb. Since the corona may change greatly within a few days-especially during the present high solar activity-the reliability of an optical corona-picture diminishes from the limb to the central meridian. In addition, a further uncertainty has to be considered in constructing coronal maps, in so far as no station possesses complete coronal observations; therefore, observations of different stations have to be used, which are very difficult to reduce to each other. The main difficulty of such a reduction arises from the fact that the single stations carry out their observations at different distances from the sun's limb ranging from 20,000 to $45,000 \mathrm{~km}$. The coronagrams discussed in. the following are based on the intensities of the green coronal line $5303 \AA$ as published in the Quarterly Bulletin on Solar Activity [2].

Fig. 1 (concerning 1957 June 27) shows in the SW-quadrant a field of strong radio emission in the surroundings of a large sunspot. This field and that of optical coronal emission are situated in somewhat higher latitudes than the spot-group. At the SW-limb there are two spot-groups in an extended region of coronal emission, whereas the radioheliogram shows a bright spot outside the solar limb. In the NW-quadrant there are three spot-groups; the two larger ones are nearer the limb and are covered by a field of enhanced radio emission. The latter lies nearer to the group in smaller heliographic latitude; whereas the maximum intensity of the optical corona lies over the 


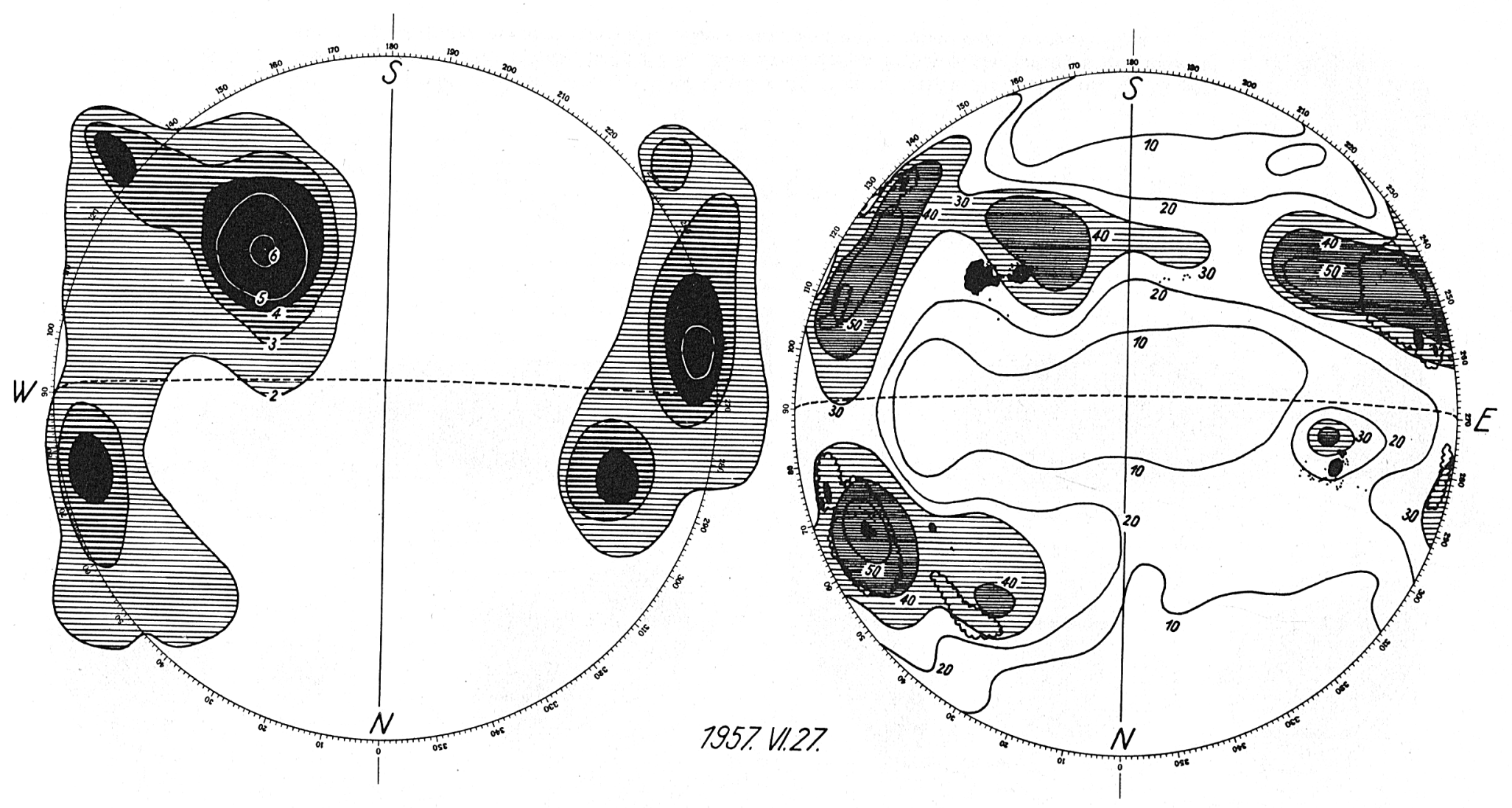

FIG. 1. Left side: Radioheliogram of 1957 June 27 . Contour brightness unit $=10^{5}{ }^{\circ} \mathrm{K}$. Right side: Isophotes of the coronal line $5303 \AA$. Brightness unit $=10^{-6} \AA$ of the spectrum of the sun's center. Spot groups are shown in black, plage areas (observed in white light) by wavy lines. 


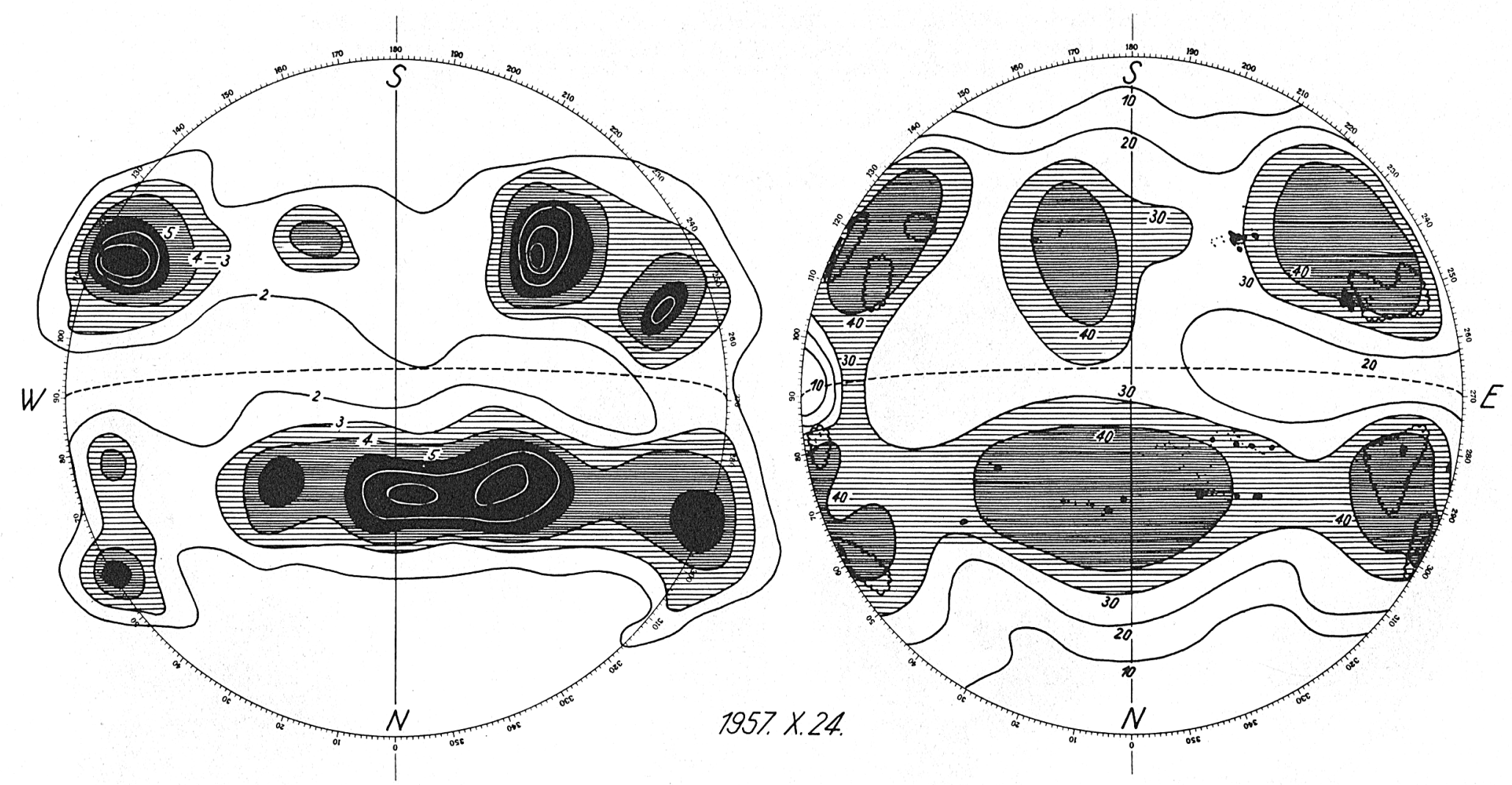

FIG. 2. Left side: Radioheliogram of 1957 October 24. Contour brightness unit $=10^{5}{ }^{\circ} \mathrm{K}$. Right side: Isophotes of the coronal line $5303 \AA$. Brightness unit $=10^{-6} \AA$ of the spectrum of the sun's center. Spot groups are shown in black, plage areas (observed in white light) by wavy lines. 
middle group. The third, smaller group does not show any maximum of radio emission, nor does the spotless field of faculae situated nearer to the central meridian and accompanied by increased coronal brightness. The NEquadrant shows an average-sized spot-group near the equator. The corresponding radio-spot lies in a somewhat greater latitude, the corresponding optical coronal emission in a somewhat smaller latitude, than the spot-group. At the SE-limb there is a rather large spot-group, whose fields of faculae and coronal emission extend to higher latitudes; the center of the radio-spot lies just over the spot-group. Finally, an isolated radio-spot exists outside the $\mathrm{SE}$-limb at 40 degrees heliographic latitude which is not connected with any optical feature.

Fig. 2 (concerning 1957 October 24) shows several spot-groups in the SWquadrant accompanied by fields of faculae and coronal emission. The optical and radio-emission regions coincide completely. A small spot-group in the SW-quadrant, not far from the central meridian, is accompanied by an extensive coronal condensation as well as by a maximum of radio emission. Likewise, near the NW-limb a close connection is observed between radio and optical emission of the corona. In the region of the northern central meridian there are numerous small spot-groups, all accompanied by a field of increased radio and optical emission. The radioheliogram presents a more detailed structure than the optical picture, which depends on the circumstance that the optical observations are made at intervals of one day, giving a resolving power of about 4 minutes of arc in the center of the solar disk, whereas the resolving power for the radioheliograms amounts to 3 minutes of arc. Near the NE-limb, two of three spot-groups, i.e. the two in higher latitudes, are accompanied by increased radio and optical emission; whereas the third group near the equator, not accompanied by enhanced radio emission, is an old spot. The two large spot-groups in the SE-quadrant are strong radio sources, whose maxima, however, are somewhat closer to the limb than to the spots. The optical emission region over these two groups again shows less structure than the radioheliogram and-with regard to the spots-the same limb-shift as the radio emission.

Recapitulating, we may confirm-as expected-that the optical coronal emission shows a better conformity than the spots and plages with the radio sources; if, however, the connection does not satisfy in every case, we have to consider that the radioheliogram presents an instantaneous picture, whereas the coronal map has to be built up from observations that are separated by several days. In addition, the optical observations are not sufficiently accurate; neither the absolute value nor the distance from the solar limb at which the measurements are carried out is known as exactly as necessary. For the future, the intensities of the coronal lines should be measured not only at one single distance but at all heights up to the disappearance of the line as has been done by the author before [3]. The present discussion is a further confirmation of the idea, first proposed by the author, that coronal condensations are the sources of the bright radio spots observed on $\mathrm{dm}$-wavelengths and of the slowly varying component [4]. 
[1] Christiansen, W. N., and Mathewson, D. S. Proc. I.R.E. 46, 127, 1958.

[2] Quarterly Bulletin on Solar Activity, Nos. 118, 120, Swiss Federal Observatory, Zürich, 1958.

[ 3 ] Waldmeier, M., Die Sonnenkorona. Basel, 1957, Vol. II, Chapter 6.

[4] Waldmeier, M., and Müller, H. Z. Ap. 27, 58, 1950.

\section{Discussion}

Vitkevich: In 1951-52 (see V. V. Vitkevich and M. Sigal in A. Zh.), I studied the nature of the slowly varying component of the sun's radio emission at wavelengths of 10 and $50 \mathrm{~cm}$. The "center of gravity" of the sun's radio emission was measured. The results of radio observations were compared with the data for the coronal regions emitting the green line ( $\lambda 5303$ ). Very good correlation was found between the data on $10 \mathrm{~cm}, 50 \mathrm{~cm}$, and $\lambda 5303$. It was concluded that coronal condensations are the regions we know as the regions of the slowly varying component. 